\title{
Achievable Rates of ZF Receivers in Massive MIMO with Phase Noise Impairments
}

\author{
Antonios Pitarokoilis, Saif Khan Mohammed and Erik G. Larsson
}

\section{Linköping University Post Print}

\section{Tweet}

N.B.: When citing this work, cite the original article.

Original Publication:

Antonios Pitarokoilis, Saif Khan Mohammed and Erik G. Larsson, Achievable Rates of ZF Receivers in Massive MIMO with Phase Noise Impairments, 2013, 2013 ASILOMAR CONFERENCE ON SIGNALS, SYSTEMS AND COMPUTERS, 1004-1008.

\section{http://dx.doi.org/}

Copyright: (C2016 IEEE. Personal use of this material is permitted. However, permission to reprint/republish this material for advertising or promotional purposes or for creating new collective works for resale or redistribution to servers or lists, or to reuse any copyrighted component of this work in other works must be obtained from the IEEE.

\section{http://ieeexplore.ieee.org/}

Postprint available at: Linköping University Electronic Press

http://urn.kb.se/resolve?urn=urn:nbn:se:liu:diva-97478 


\title{
Achievable Rates of ZF Receivers in Massive MIMO with Phase Noise Impairments
}

\author{
Antonios Pitarokoilis $^{1}$, Saif Khan Mohammed ${ }^{2}$, Erik G. Larsson ${ }^{1}$ \\ ${ }^{1}$ Dept. of Electrical Engineering (ISY), Linköping University, Linköping, Sweden, \{antonispit, egl $\} @$ isy.liu.se \\ 2 Dept. of Electrical Engineering, Indian Institute of Technology (I.I.T.) Delhi, India, saifkm@ee.iitd.ac.in
}

\begin{abstract}
The effect of oscillator phase noise on the sum-rate performance of large multi-user multiple-input multiple-output (MU-MIMO) systems is studied. A Rayleigh fading MU-MIMO uplink channel is considered, where channel state information (CSI) is acquired via training. The base station (BS), which is equipped with an excess of antenna elements, $M$, uses the channel estimate to perform zero-forcing (ZF) detection. A lower bound on the sum-rate performance is derived. It is shown that the proposed receiver structure exhibits an $O(\sqrt{M})$ array power gain. Additionally, the proposed receiver is compared with earlier studies that employ maximum ratio combining and it is shown that it can provide significant sum-rate performance gains at the medium and high signal-to-noise-ratio (SNR) regime. Further, the expression of the achievable sum rate provides new insights on the effect of various parameters on the overall system performance.
\end{abstract}

\section{INTRODUCTION}

In the last decades there has been an unprecedented increase in demand for high data rates over wireless cellular systems. Multiple-input multiple-output (MIMO) systems have attracted significant attention since they promise substantial gains in the capacity of wireless networks [1], [2]. Such systems have gained popularity and are already part of modern cellular communication standards. The demand for increased high speed wireless access is expected to accelerate in the years to come. In addition, power consumption at the base stations (BS) of cellular systems is a major problem. Therefore, future cellular systems are required to exhibit increased spectral efficiency as well as improved energy efficiency.

Recently, it has been shown that using an excess of BS antenna elements, $M$, (Massive MIMO) can boost data rates and simultaneously it can reduce the required transmit power with very simple linear signal processing [3], [4]. In [5] the authors show that for the case of MU-MIMO uplink channel with linear receivers, one can reduce the total transmitted power proportionally to $M$ (or $\sqrt{M}$ ) for the case of perfect (or imperfect) channel state information (CSI), without compromising the total throughput. Further studies also highlight the gains in energy efficiency that are offered by Massive MIMO [6], [7].

In practice, communication systems inevitably suffer from transceiver impairments. One such impairment is phase noise. Phase noise is introduced by imperfect oscillators that convert the baseband signal to the passband and vice versa. Ideal oscillators output a sinusoid that is stable in amplitude, frequency and phase. However, due to imperfections of the circuitry of the oscillators the phase of the sinusoid drifts

This work was supported by the Swedish Foundation for Strategic Research (SSF) and ELLIIT. randomly. Consequently, phase noise can significantly degrade the performance of coherent communication. In a system where CSI is acquired via uplink training, phase noise causes a partial loss of coherency between the estimated channel coefficients and the channel realizations during detection. The large array gain offered by Massive MIMO is a consequence of the coherent combining of received signals using the estimated channel gains. Therefore, the study of the effect of phase noise impairment on the performance of Massive MIMO systems is particularly important.

In earlier work [8], we have studied the effect of oscillator phase noise in the frequency selective Massive MIMO uplink when detection is performed via time-reversal maximum-ratiocombining (TR-MRC). TR-MRC is optimal at low signal-tonoise-ratio (SNR), where the system performance is limited by thermal noise. However, in the high-SNR regime the system performance is interference limited and the MRC sum-rate performance saturates. Zero-forcing $(\mathrm{ZF})$ receivers can provide improved performance in this regime since they effectively suppress multi-user interference. In this work we study the sum-rate performance of $\mathrm{ZF}$ receivers with phase noise impairments and compare it with the sum-rate performance of MRC receivers. To the best of authors' knowledge, this paper is the first to report such a study.

We consider a Rayleigh fading channel with $K$ noncooperative users and $M$ BS antennas. Phase noise is introduced both at the transmitter and receiver side and is modelled as a Wiener phase noise process. The BS estimates the channel via uplink training and uses the acquired CSI to perform $\mathrm{ZF}$ detection. We provide an analytical formula for a lower bound on the sum-capacity and a coding strategy that achieves this lower bound. We prove that, just like MRC, ZF also offers an $O(\sqrt{M})$ array power gain. We show that $\mathrm{ZF}$ is superior to MRC in term of sum-rate performance in the high-SNR regime. For a desired per-user information rate, we also study the extra per-user transmit power required by MRC receivers when compared to the $\mathrm{ZF}$ receiver. The derived achievable sum-rate can provide new insights in the design of Massive MIMO systems with phase noise impairments.

\section{SySTEM MODEL}

We consider a frequency flat multi-user multiple-input multiple-output (MU-MIMO) uplink channel, with $K$ single antenna users and $M$ base station (BS) antenna elements. The channel between user $k$ and antenna element $m$ is given by $\sqrt{d_{k}} h_{m, k}$, where $d_{k}$ and $h_{m, k}$ models the slow and fast fading components, respectively. In this work, we assume 
a block fading model, where $h_{m, k}$ is fixed for a block of $K+N_{D}$ channel uses and varies independently from one block to another. Since $d_{k}$ changes at a much slower rate compared to $h_{m, k}$, we assume that it is fixed and known throughout the communication. The fast fading coefficients $h_{m, k}$ are assumed to be independent identically distributed (i.i.d.) circularly symmetric complex normal $\mathcal{C N}(0,1)$ random variables.

Phase noise is introduced at the transmitter during upconversion, when the baseband signal is multiplied with the carrier generated by the oscillator. The phase of the generated carrier drifts randomly, resulting in phase distortion of the transmitted signal. A similar phenomenon also happens at the receiver side during down-conversion of the bandpass signal to baseband. In the following, $\theta_{k}, k=1, \ldots, K$ denotes the phase noise process at the $k$-th user and $\phi$ denotes the phase noise process at the $M$ BS receivers. The latter implies identical phase noise processes at the BS antenna elements, i.e. we assume full coherency between the $M$ BS receivers. This models the practical scenario of a centralized BS with a single oscillator output feeding the down-conversion module in each receiver. We further assume that the phase noise processes $\phi, \theta_{k}, k=1, \ldots, K$ are mutually independent. In this study every phase noise process is modelled as an independent Wiener process, which is a well-established model [9], [10]. Therefore, the discrete time phase noise process at the BS antennas at time $n$ is given by ${ }^{1} \phi[i]=\phi[i-1]+w[i]$, where $w[i] \sim \mathcal{N}\left(0, \sigma_{\phi}^{2}\right), \sigma_{\phi}^{2} \triangleq 4 \pi^{2} f_{c}^{2} c T_{s}$ are independent identically distributed zero-mean Gaussian increments. $f_{c}$ is the carrier frequency, $T_{s}$ is the symbol interval and $c$ is a constant that depends on the oscillator. Similarly, we can define the phase noise processes at the $K$ users.

Let $x_{k}[i]$ be the symbol transmitted from the $k$-th user at time $i$. The received signal at the $m$-th BS antenna element at time $i$ is then given by

$$
y_{m}[i]=\sqrt{P} \sum_{k=1}^{K} \sqrt{d_{k}} h_{m, k} e^{-j\left(\phi[i]-\theta_{k}[i]\right)} x_{k}[i]+n_{m}[i],
$$

where $n_{m}[i] \sim \mathcal{C N}\left(0, \sigma^{2}\right)$ is additive white Gaussian noise (AWGN). Each user transmits a stream of i.i.d. $\mathcal{C N}(0,1)$ information symbols (i.e. $x_{k}[i] \sim \mathcal{C N}(0,1)$ ), that are independent of the information symbols of the other users. $P$ denotes the average uplink transmitted power from each user.

\section{TRANSMISSION SCHEME AND ZF RECEIVER}

Motivated by the need for low-complexity channel estimation and detection algorithms, we propose the following block based uplink transmission scheme. In the proposed scheme, a transmission block of $K+N_{D}$ channel uses consists of $K$ channel uses (for uplink channel estimation) followed by the data phase (for data transmission) of duration $N_{D}$ channel uses. For coherent demodulation, the BS needs to estimate the

\footnotetext{
${ }^{1}$ The discrete-time phase noise model is used since we are interested in the discrete-time complex baseband representation of the transmit and receive signals.
}

uplink channel. This is facilitated through the transmission of uplink pilot symbols during the training phase of each transmission block. The users transmit uplink training signals sequentially in time, i.e. at any given time only one user is transmitting uplink training signals and all other users are idle. To be precise, the $k$-th user sends an impulse signal of amplitude $\sqrt{P_{p} K}$ at the $(k-1)$-th channel use and is idle for the remaining portion of the training phase. Here, $P_{p}$ is the average power transmitted by a user during the training phase. Therefore, using (1) the signal received at the $m$-th BS receiver at time $k-1, k=1, \ldots, K$ is given by

$y_{m}[k-1]=\sqrt{P_{p} K d_{k}} h_{m, k} e^{-j\left(\phi[k-1]-\theta_{k}[k-1]\right)}+n_{m}[k-1]$.

\section{A. LMMSE Channel Estimation}

Based on the received signal during training, the effective MIMO channel is estimated in order to enable coherent detection of the received symbols. The received symbols during training, given by (2), can be expressed in the following matrix-vector form

$$
\boldsymbol{Y}=\sqrt{P_{p} K} \boldsymbol{H} \boldsymbol{\Omega} \boldsymbol{D}^{1 / 2}+\boldsymbol{N}
$$

where $[\boldsymbol{Y}]_{m, k}=y_{m}[k-1],[\boldsymbol{H}]_{m, k}=h_{m, k}$, $\boldsymbol{\Omega}=\operatorname{diag}\left\{e^{-j\left(\phi[0]-\theta_{1}[0]\right)}, \cdots, e^{-j\left(\phi[K-1]-\theta_{K}[K-1]\right)}\right\}, \boldsymbol{D}=$ $\operatorname{diag}\left\{d_{1}, \ldots, d_{K}\right\}, d_{k}>0, \forall k=1, \ldots, K$ and $[\boldsymbol{N}]_{m, k}=$ $n_{m}[k-1]$. Also, observe that $\boldsymbol{\Omega} \boldsymbol{\Omega}^{H}=\boldsymbol{\Omega}^{H} \boldsymbol{\Omega}=\boldsymbol{I}_{K}$. We define the effective MIMO channel matrix as $\boldsymbol{G} \triangleq \boldsymbol{H} \boldsymbol{\Omega}$. The LMMSE estimate $\hat{\boldsymbol{G}}$ for $\boldsymbol{G}$ based on (3) is given by [11]

$$
\hat{\boldsymbol{G}}=\boldsymbol{Y} \operatorname{diag}\left\{\frac{\sqrt{P_{p} K d_{1}}}{P_{p} K d_{1}+\sigma^{2}}, \ldots, \frac{\sqrt{P_{p} K d_{K}}}{P_{p} K d_{K}+\sigma^{2}}\right\} .
$$

As expected, the channel estimate is distorted by the AWGN and by the phase noise at the transmitter and at the BS. In the following, we treat this channel estimate as the true channel and perform equalization based on that. Conditioned on $\Omega$, it is clear from (3) that the entries of $\boldsymbol{Y}$ are Gaussian distributed. Since $\boldsymbol{G}=\boldsymbol{H} \boldsymbol{\Omega}$ and $\hat{\boldsymbol{G}}$ is a column wise scaled version of $\boldsymbol{Y}$ (see (4)), it follows that conditioned on $\Omega, G, \hat{G}$ and $\mathcal{E} \triangleq$ $\hat{\boldsymbol{G}}-\boldsymbol{G}$ are all Gaussian distributed. Therefore, conditioned on $\Omega$, the LMMSE estimate in (4) is actually the MMSE estimate and therefore $\hat{G}$ and $\mathcal{E}$ are independent (conditioned on $\Omega$ ). Since we are interested in evaluating the performance of $\mathrm{ZF}$ receivers, the equalizer would simply be the pseudo-inverse of $\hat{\boldsymbol{G}}$, i.e. $\hat{G}^{\dagger} \triangleq\left(\hat{\boldsymbol{G}}^{H} \hat{\boldsymbol{G}}\right)^{-1} \hat{\boldsymbol{G}}^{H}$. Since $\hat{\boldsymbol{G}}$ and $\mathcal{E}$ are independent (conditioned on $\Omega$ ), it therefore follows that

$$
\mathbb{E}_{\hat{\boldsymbol{G}}, \mathcal{E} \mid \boldsymbol{\Omega}}\left[\hat{\boldsymbol{G}}^{\dagger} \mathcal{E}\right]=\mathbb{E}_{\hat{\boldsymbol{G}} \mid \boldsymbol{\Omega}}\left[\hat{\boldsymbol{G}}^{\dagger}\right] \mathbb{E}_{\mathcal{E} \mid \boldsymbol{\Omega}}[\mathcal{E}]=0
$$

since $\mathbb{E}_{\mathcal{E} \mid \Omega}[\mathcal{E}]=0$.

\section{B. Zero-Forcing $(\mathrm{ZF})$ Equalization}

During the data interval, the received symbol at time $i \geq K$ at the $m$-th $\mathrm{BS}$ antenna is given by

$$
y_{m}[i]=\sqrt{P_{D}} \sum_{k=1}^{K} h_{m, k} e^{-j\left(\phi[i]-\theta_{k}[i]\right)} \sqrt{d_{k}} x_{k}[i]+n_{m}[i] .
$$


Therefore, the matrix-vector form of the channel input-output relation is given by

$$
\boldsymbol{y}[i]=\sqrt{P_{D}} \boldsymbol{H} \boldsymbol{\Phi}[i] \boldsymbol{D}^{1 / 2} \boldsymbol{x}[i]+\boldsymbol{n}[i],
$$

where $\boldsymbol{\Phi}[i] \triangleq \operatorname{diag}\left\{e^{-j\left(\phi[i]-\theta_{1}[i]\right)}, \ldots, e^{-j\left(\phi[i]-\theta_{K}[i]\right)}\right\}$ and $P_{D}$ is the per-user average transmit power constraint during the data interval. In general, the relation between $P_{D}$ and $P_{p}$ can be arbitrary. However, in this work we restrict to the case where $P_{p}=\beta P_{D}, \beta>0$.

Since $\boldsymbol{G}=\boldsymbol{H} \boldsymbol{\Omega}$ and $\boldsymbol{\Omega} \boldsymbol{\Omega}^{H}=\boldsymbol{I}_{K}$, it follows that $\boldsymbol{H}=$ $\boldsymbol{G} \boldsymbol{\Omega}^{H}$. Also, since $\boldsymbol{G}=\hat{\boldsymbol{G}}-\mathcal{E}$, we have $\boldsymbol{H}=\boldsymbol{G} \boldsymbol{\Omega}^{H}=$ $(\hat{\boldsymbol{G}}-\mathcal{E}) \boldsymbol{\Omega}^{H}$. Therefore, replacing $\boldsymbol{H}$ by $(\hat{\boldsymbol{G}}-\mathcal{E}) \boldsymbol{\Omega}^{H}$ in (7) we get

$$
\boldsymbol{y}[i]=\sqrt{P_{D}}(\hat{\boldsymbol{G}}-\mathcal{E}) \boldsymbol{\Omega}^{H} \boldsymbol{\Phi}[i] \boldsymbol{D}^{1 / 2} \boldsymbol{x}[i]+\boldsymbol{n}[i] .
$$

Then, the detected symbol vector $\hat{\boldsymbol{x}}[i]$ at time $i$ is given by

$$
\begin{aligned}
\hat{\boldsymbol{x}}[i]=\hat{\boldsymbol{G}}^{\dagger} \boldsymbol{y}[i] & =\sqrt{P_{D}} \boldsymbol{\Omega}^{H} \boldsymbol{\Phi}[i] \boldsymbol{D}^{1 / 2} \boldsymbol{x}[i] \\
& -\sqrt{P_{D}} \hat{\boldsymbol{G}}^{\dagger} \mathcal{E} \boldsymbol{\Omega}^{H} \boldsymbol{\Phi}[i] \boldsymbol{D}^{1 / 2} \boldsymbol{x}[i]+\hat{\boldsymbol{G}}^{\dagger} \boldsymbol{n}[i],
\end{aligned}
$$

where $\hat{\boldsymbol{G}}^{\dagger} \triangleq\left(\hat{\boldsymbol{G}}^{H} \hat{\boldsymbol{G}}\right)^{-1} \hat{\boldsymbol{G}}^{H}$ is the pseudo-inverse of $\hat{\boldsymbol{G}}$.

\section{Achievable Rates}

In this work we are interested in evaluating the sum-rate performance for the system under study. In the following, we describe the approach to derive an achievable sum rate for the Massive MIMO uplink with phase noise impairments when $\mathrm{ZF}$ reception is employed. From (9) the detected symbol for the $k$-th user at time $i$ is given by

$$
\begin{aligned}
\hat{x}_{k}[i] & =\sqrt{P_{D} d_{k}} e^{j\left(\phi[k-1]-\theta_{k}[k-1]\right)} e^{-j\left(\phi[i]-\theta_{k}[i]\right)} x_{k}[i] \\
& -\sqrt{P_{D}} \boldsymbol{e}_{k}^{T} \hat{\boldsymbol{G}}^{\dagger} \mathcal{E} \boldsymbol{\Omega}^{H} \boldsymbol{\Phi}[i] \boldsymbol{D}^{1 / 2} \boldsymbol{x}[i]+\boldsymbol{e}_{k}^{T} \hat{\boldsymbol{G}}^{\dagger} \boldsymbol{n}[i],
\end{aligned}
$$

where $\boldsymbol{e}_{k}$ is the $K$-dimensional all-zero column vector that has a single 1 at position $k$. Let $A_{k}[i] \triangleq$ $\sqrt{P_{D} d_{k}} e^{j\left(\phi[k-1]-\theta_{k}[k-1]\right)} e^{-j\left(\phi[i]-\theta_{k}[i]\right)}$. Since the exact phase noise realizations are unknown, the desired symbol $x_{k}[i]$ is rotated by an unknown phase. Further, the statistics of $A_{k}[i]$ depends on $i$. However, the statistics of the phase noise processes is known to the BS and as a result it knows the mean value of $A_{k}[i]$. In (10) we add and subtract the term $\mathbb{E}\left[A_{k}[i]\right] x_{k}[i]$. The added term $\mathbb{E}\left[A_{k}[i]\right] x_{k}[i]$ is the desired signal term and the term $\left(A_{k}[i]-\mathbb{E}\left[A_{k}[i]\right]\right) x_{k}[i]$ is relegated to an effective noise term. Therefore, (10) can be expressed as

$$
\hat{x}_{k}[i]=\mathbb{E}\left[A_{k}[i]\right] x_{k}[i]+\mathrm{EN}_{k}[i]
$$

where

$$
\begin{aligned}
\operatorname{EN}_{k}[i] & \triangleq \operatorname{IF}_{k}[i]+\operatorname{MUI}_{k}[i]+\operatorname{AN}_{k}[i] \\
\operatorname{IF}_{k}[i] & \triangleq\left(A_{k}[i]-\mathbb{E}\left[A_{k}[i]\right]\right) x_{k}[i], \\
\operatorname{MUI}_{k}[i] & \triangleq-\sqrt{P_{D}} \boldsymbol{e}_{k}^{T} \hat{\boldsymbol{G}}^{\dagger} \mathcal{E} \boldsymbol{\Omega}^{H} \boldsymbol{\Phi}[i] \boldsymbol{D}^{1 / 2} \boldsymbol{x}[i], \\
\mathrm{AN}_{k}[i] & \triangleq \boldsymbol{e}_{k}^{T} \hat{\boldsymbol{G}}^{\dagger} \boldsymbol{n}[i] .
\end{aligned}
$$

Proposition 1: In (11) the desired signal term $\mathbb{E}\left[A_{k}[i]\right] x_{k}[i]$ and the effective additive noise term $\operatorname{EN}_{k}[i]$ are uncorrelated.

Proof: It is straightforward to see that $\mathbb{E}\left[\mathbb{E}\left[A_{k}[i]\right] x_{k}[i] \mathrm{IF}_{k}^{*}[i]\right]=0$. Also $\mathbb{E}\left[\mathbb{E}\left[A_{k}[i]\right] x_{k}[i] \mathrm{AN}_{k}^{*}[i]\right]=$ 0 by the assumption that $\boldsymbol{x}[i]$ and $\boldsymbol{n}[i]$ are uncorrelated. Finally,

$$
\begin{aligned}
& \mathbb{E}\left[\operatorname{MUI}_{k}[i] x_{k}^{*}[i] \mathbb{E}\left[A_{k}^{*}[i]\right]\right]=-\sqrt{P_{D}} \mathbb{E}\left[\boldsymbol{e}_{k}^{T} \hat{\boldsymbol{G}}^{\dagger} \mathcal{E} \boldsymbol{\Omega}^{H} \boldsymbol{\Phi}[i] \boldsymbol{D}^{1 / 2} \boldsymbol{e}_{k}\right] \\
& \cdot \mathbb{E}\left[A_{k}^{*}[i]\right]=-\sqrt{P_{D} d_{k}} \mathbb{E}_{\boldsymbol{\Omega}}\left[\boldsymbol{e}_{k}^{T} \mathbb{E}_{\hat{\boldsymbol{G}}, \boldsymbol{\mathcal { E }} \mid \boldsymbol{\Omega}}\left[\hat{\boldsymbol{G}}^{\dagger} \mathcal{E}\right] \boldsymbol{e}_{k} e^{-j\left(\phi[i]-\theta_{k}[i]\right)}\right. \\
& \left.\cdot e^{j\left(\phi[k-1]-\theta_{k}[k-1]\right)}\right] \mathbb{E}\left[A_{k}^{*}[i]\right]=0,
\end{aligned}
$$

which follows since $\mathbb{E}_{\hat{G}, \mathcal{E} \mid \boldsymbol{\Omega}}\left[\hat{\boldsymbol{G}}^{\dagger} \mathcal{E}\right]=0$ from (5). Hence, we can conclude that $\mathbb{E}\left[\mathbb{E}\left[A_{k}[i] x_{k}[i] \mathrm{EN}_{k}^{*}[i]\right]=0\right.$.

We also provide a result that will be proved useful in the derivation of the achievable rates.

Theorem 1: The mean value of $A_{k}[i]$ and the variance $\operatorname{Var}\left(\operatorname{EN}_{k}[i]\right) \triangleq \mathbb{E}\left[\left|\operatorname{EN}_{k}[i]-\mathbb{E}\left[\operatorname{EN}_{k}[i]\right]\right|^{2}\right]$ are given by

$$
\mathbb{E}\left[A_{k}[i]\right]=\sqrt{P_{D} d_{k}} \kappa_{k}[i],
$$

$$
\operatorname{Var}\left(\operatorname{EN}_{k}[i]\right)=P_{D} d_{k}\left(1-\kappa_{k}^{2}[i]\right)+\sigma^{2} C_{k},
$$

where $\kappa_{k}[i]=e^{-\sigma_{\phi}^{2}(i-(k-1))}$ and

$$
C_{k} \triangleq\left(\sum_{l=1}^{K} \frac{\frac{P_{D}}{\sigma^{2}} d_{l}}{\beta \frac{P_{D}}{\sigma^{2}} K d_{l}+1}+1\right) \frac{\beta \frac{P_{D}}{\sigma^{2}} K d_{k}+1}{(M-K) \beta \frac{P_{D}}{\sigma^{2}} K d_{k}} .
$$

In the following, we describe a method to derive an achievable information rate for the $k$-th user. Similar techniques have been used earlier in [12], [13], [8]. Observe that, in a given coherence interval, $\mathbb{E}\left[A_{k}[i]\right]$ and $\operatorname{Var}\left(\operatorname{EN}_{k}[i]\right)$ are different for different $i$. However $\mathbb{E}\left[A_{k}[i]\right]$ and $\operatorname{Var}\left(\mathrm{EN}_{k}[i]\right)$ for a fixed $k$ and $i$ are the same across multiple coherence intervals. Further, the realizations of $\mathrm{EN}_{k}[i]$ for a fixed $i$ and $k$ are i.i.d. across multiple coherence intervals. Therefore, for the $k$-th user, we propose to encode information using $N_{D}$ different channel codes, one channel code for each $i$. That is, in the $i$-th channel code information is coded across the $i$-th channel use of each coherence interval.

We proceed by describing the derivation of a lower bound for the rate of the $i$-th channel code for the $k$-th user. Since the BS has knowledge of the statistics of the channel coefficients and the phase noise processes but not of the exact realization, the term $\mathbb{E}\left[A_{k}[i]\right]$ can be computed and is a constant depending on the user $k$ and the channel use $i$ during the data transmission interval. On the other hand, the exact probability distribution of the effective noise term is complicated to compute. However, since the input symbols are uncorrelated to the effective noise $\mathrm{EN}_{k}[i]$ (from Proposition 1), a lower bound on the achievable information rate can be derived by considering the worst case uncorrelated noise having the same variance as $\mathrm{EN}_{k}[i]$. Given that the input symbols are Gaussian, the worst case uncorrelated additive noise is also zero mean Gaussian with variance equal to the variance of $\mathrm{EN}_{k}[i]$. Therefore the mutual information between 
the detected symbol $\hat{x}_{k}[i]$ and the transmitted symbol is lower bounded by

$$
I\left(\hat{x}_{k}[i] ; x_{k}[i]\right) \geq \log _{2}\left(1+\frac{\left(\mathbb{E}\left[A_{k}[i]\right]\right)^{2}}{\operatorname{Var}\left(\operatorname{EN}_{k}[i]\right)}\right) .
$$

Theorem 2: An achievable rate for the $i$-th channel code of the $k$-th user is given by

$$
R_{k}^{\mathrm{ZF}}[i]=\log _{2}\left(1+\frac{\frac{P_{D}}{\sigma^{2}} d_{k} \kappa_{k}^{2}[i]}{\frac{P_{D}}{\sigma^{2}} d_{k}\left(1-\kappa_{k}^{2}[i]\right)+C_{k}}\right)
$$

Proof: The result follows directly by substituting (12) and (13) into (14).

The information rate for the no-phase noise case (i.e. $c=0$, perfect oscillator) can be derived by substituting $\kappa_{k}[i]=1$ in 15 , and is given by

$\mathcal{R}_{k}=\log _{2}\left(1+\frac{\beta K(M-K)\left(\frac{P_{D}}{\sigma^{2}}\right)^{2} d_{k}^{2}}{\left(\beta \frac{P_{D}}{\sigma^{2}} K d_{k}+1\right)\left(\sum_{l=1}^{K} \frac{\frac{P_{D}}{\sigma^{2}} d_{l}}{\beta \frac{P_{D}}{\sigma^{2}} K d_{l}+1}+1\right)}\right)$

For the sake of comparison, we also derive the information rate expression for maximum-ratio-combining (MRC) when MMSE channel estimation is used (using similar arguments to the $\mathrm{ZF}$ case). The $i$-th code of user $k$ achieves the rate

$$
R_{k}^{\mathrm{MRC}}[i]=\log _{2}\left(1+\frac{\frac{P_{D}}{\sigma^{2}} d_{k} \kappa_{k}^{2}[i]}{\frac{P_{D}}{\sigma^{2}} d_{k}\left(1-\kappa_{k}^{2}[i]\right)+C_{k}^{\mathrm{MRC}}}\right),
$$

where $C_{k}^{\mathrm{MRC}} \triangleq \frac{\frac{\beta P_{D}}{\sigma^{2}} K d_{k}+1}{\frac{\beta P_{D}}{\sigma^{2}} M K d_{k}}\left(\frac{P_{D}}{\sigma^{2}} \sum_{l=1}^{K} d_{l}+1\right)$. We conclude the section by defining the average achievable sum-rate for ZF as

$$
R^{\mathrm{ZF}} \triangleq \frac{1}{N_{D}+K} \sum_{k=1}^{K} \sum_{i=K}^{N_{D}+K-1} R_{k}^{\mathrm{ZF}}[i],
$$

where the normalization factor $\frac{1}{N_{D}+K}$ accounts for the loss of spectral efficiency due to training. Similarly, one can define the average achievable sum-rate for the MRC and no-phase noise cases.

\section{RESUlts - Discussion}

In this section we use the expressions derived in (15), (16), (17) and (18) to present our main results. In the plots we always consider $\boldsymbol{D}=\boldsymbol{I}_{K}$, which corresponds to the case of equal path loss for all the users, whereas in the theoretical derivations we consider an arbitrary, known and deterministic slow fading matrix $\boldsymbol{D}$. We start with a proposition on the information rate achievable at high SNR.

Proposition 2: In the high-SNR regime the rate that user $k$ can achieve at the $i$-th channel use saturates to the value

$$
\lim _{\frac{P D}{\sigma^{2}} \rightarrow \infty} R_{k}^{\mathrm{ZF}}[i] \rightarrow \log _{2}\left(1+\frac{\kappa_{k}^{2}[i]}{1-\kappa_{k}^{2}[i]}\right)
$$

for $\mathrm{ZF}$ and at the value

$$
\lim _{\frac{P_{D}}{\sigma^{2}} \rightarrow \infty} R_{k}^{\mathrm{MRC}}[i] \rightarrow \log _{2}\left(1+\frac{d_{k} \kappa_{k}^{2}[i]}{d_{k}\left(1-\kappa_{k}^{2}[i]\right)+\frac{1}{M} \sum_{l=1}^{K} d_{l}}\right)
$$

for MRC.

A desirable property of Massive MIMO is the array power gain they offer, facilitating the design of power efficient communication systems. In earlier work [5], [8] it has been shown that in the case of Massive MIMO with imperfect CSI, one can scale down the total transmit power by $\sqrt{M}$ as the number of BS antennas $M$ increase, while maintaining a fixed positive desired information rate to each user. In this work, we extend the result to the case of Massive MIMO uplink impaired with phase noise when $\mathrm{ZF}$ receivers are employed.

Proposition 3: An $O(\sqrt{M})$ array power gain is achievable. This implies that a fixed non-zero per user information rate can be achieved if the total transmitted power is reduced by $1.5 \mathrm{~dB}$ and at the same time the number of BS antenna elements is doubled.

Proof: From (15) and by the substitution $P_{D}=\frac{E_{u}}{M^{\alpha}}, \alpha>$ 0 we have that

$$
R_{k}^{\mathrm{ZF}}[i]=\log _{2}\left(1+\frac{\frac{E_{u}}{\sigma^{2}} d_{k} \kappa_{k}^{2}[i]}{\frac{E_{u}}{\sigma^{2}} d_{k}\left(1-\kappa_{k}^{2}[i]\right)+M^{\alpha} C_{k}}\right) .
$$

We look for the largest $\alpha>0$ such that $\lim _{M \rightarrow \infty} R_{k}[i] \rightarrow$ $r>0 \Leftrightarrow \lim _{M \rightarrow \infty} M^{\alpha} C_{k} \rightarrow$ const.

$$
\begin{aligned}
M^{\alpha} C_{k} & =M^{\alpha}\left(\sum_{l=1}^{K} \frac{\frac{E_{u}}{\sigma^{2} M^{\alpha}} d_{l}}{\beta \frac{E_{u}}{\sigma^{2} M^{\alpha}} K d_{l}+1}\right) \frac{\beta K \frac{E_{u}}{\sigma^{2} M^{\alpha}} d_{k}+1}{(M-K) \beta \frac{E_{u}}{\sigma^{2} M^{\alpha}} K d_{k}} \\
& +M^{\alpha} \frac{\beta K \frac{E_{u}}{\sigma^{2} M^{\alpha}} d_{k}+1}{(M-K) \beta \frac{E_{u}}{\sigma^{2} M^{\alpha}} K d_{k}} \\
& =\left(\sum_{l=1}^{K} \frac{\frac{E_{u}}{\sigma^{2}} d_{l} M^{\alpha}}{\beta \frac{E_{u}}{\sigma^{2}} K d_{l}+M^{\alpha}}\right) \frac{\beta K \frac{E_{u}}{\sigma^{2}} d_{k}+M^{\alpha}}{(M-K) \beta \frac{E_{u}}{\sigma^{2}} K d_{k}} \\
& +\frac{\beta K \frac{E_{u}}{\sigma^{2}} d_{k}+M^{\alpha}}{\frac{(M-K)}{M^{\alpha}} \beta \frac{E_{u}}{\sigma^{2}} K d_{k}}
\end{aligned}
$$

As $M \rightarrow \infty$, the first term converges to a finite positive constant if and only $\alpha \leq 1$, whereas the second term converges to a finite positive constant if and only if $\alpha \leq 1 / 2$. For $\alpha>1 / 2$, the second term is unbounded. Therefore, as $M \rightarrow \infty$, with $P_{D}=\frac{E_{u}}{M^{\alpha}}$, we have

$$
\lim _{M \rightarrow \infty} R_{k}^{\mathrm{ZF}}[i] \rightarrow \begin{cases}\log _{2}\left(1+\frac{\frac{E_{u}}{\sigma^{2}} d_{k} \kappa_{k}^{2}[i]}{\left.\frac{E_{u}}{\sigma^{2}} d_{k}\left(1-\kappa_{k}^{2}[i]\right)+\frac{1}{\beta \frac{E_{u} K d_{k}}{\sigma^{2}}}\right),}\right. & \alpha=1 / 2 \\ \log _{2}\left(1+\frac{\kappa_{k}^{2}[i]}{1-\kappa_{k}^{2}[i]}\right), & \alpha<1 / 2 \\ 0, & \alpha>1 / 2\end{cases}
$$

In Fig. 1 we plot the minimum required $\frac{P_{D}}{\sigma^{2}}$ to achieve a fixed desired per-user information rate $r=1.5 \mathrm{bpcu}$ as a function of the number of BS antennas $M$ for fixed $K=10$ users, $N_{D}=100$ channel uses, $T_{s}=10^{-6} \mathrm{~s}$ and $c=4.7 \times$ $10^{-18}(\mathrm{rad} \mathrm{Hz})^{-1}$. We plot the curves for ZF and MRC with 


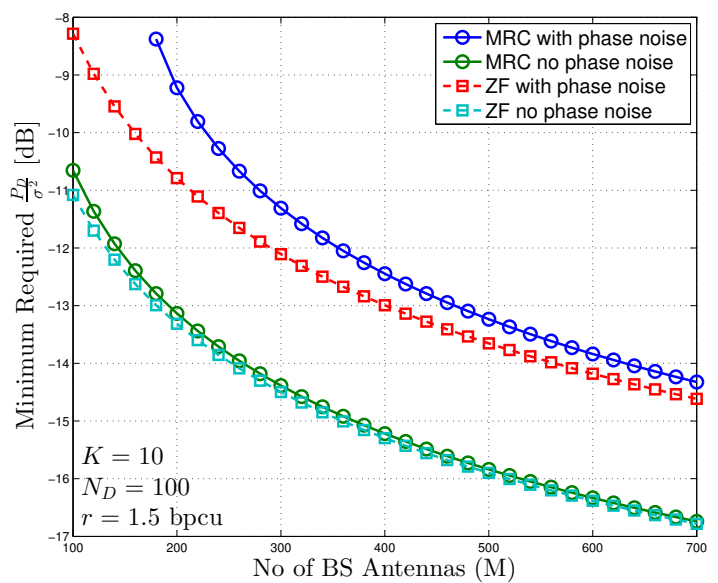

Fig. 1. Minimum required $\frac{P_{D}}{\sigma^{2}}$ to achieve a fixed per-user information rate of $r=1.5 \mathrm{bpcu}$ as a function of increasing $M$ for fixed $K=10$ users, $N_{D}=100$ channel uses, $T_{s}=10^{-6} \mathrm{~s}$ and $c=4.7 \times 10^{-18}(\mathrm{rad} \mathrm{Hz})^{-1}$.

and without phase noise. It is clear that at large $M$, when the number of BS antennas doubles the minimum required power decreases by $1.5 \mathrm{~dB}$. This verifies the $O(\sqrt{M})$ array power gain as stated in Proposition 3.

Further, the superior performance of the $\mathrm{ZF}$ over MRC in the high-SNR regime is clear. When it is desired to achieve a per-user rate of $r=1.5 \mathrm{bpcu}$ with $\frac{P_{D}}{\sigma^{2}}=-10 \mathrm{~dB}, \mathrm{ZF}$ with phase noise requires the use of about $M=160 \mathrm{BS}$ antennas. However, the MRC system with phase noise for the same parameters requires approximately 80 additional BS antennas. This motivates us to study the $\frac{P_{D}}{\sigma^{2}}$ gap between $\mathrm{ZF}$ and MRC reception. In Fig. 2 we plot this gap as a function of the desired per-user information rate for fixed $M=200$ BS antennas and $N_{D}=150$ channel uses for various values of the number of users, $K$. It is clear that there is a significant gain in the minimum required power for per-user information rates larger than $1 \mathrm{bpcu}$. The gain is more pronounced as the number of users increases. The reason for this behaviour is that for $r>1 \mathrm{bpcu}$ and for $K$ sufficiently large, MRC is limited by interference, whereas the $\mathrm{ZF}$ receiver effectively suppresses multi-user interference yielding better performance. At a peruser rate of $r \approx 0.9 \mathrm{bpcu}$ there is a turning point. Below this value we operate in the low spectral efficiency regime and the system is limited by the thermal noise. As a result MRC exhibits better performance whereas $\mathrm{ZF}$ underutilizes the available degrees of freedom.

In conclusion, we studied the effect of oscillator phase noise in a frequency flat Massive MIMO uplink channel when ZF equalization is employed at the BS. We provided an analytical expression on the achievable sum-rate and we described a coding strategy that achieves the derived sum-rate. Based on the derived expression we proved that an $O(\sqrt{M})$ array power gain is still attainable. Further, we showed that even in the presence of phase noise, ZF equalization performs significantly better than MRC at high spectral efficiencies. The derived

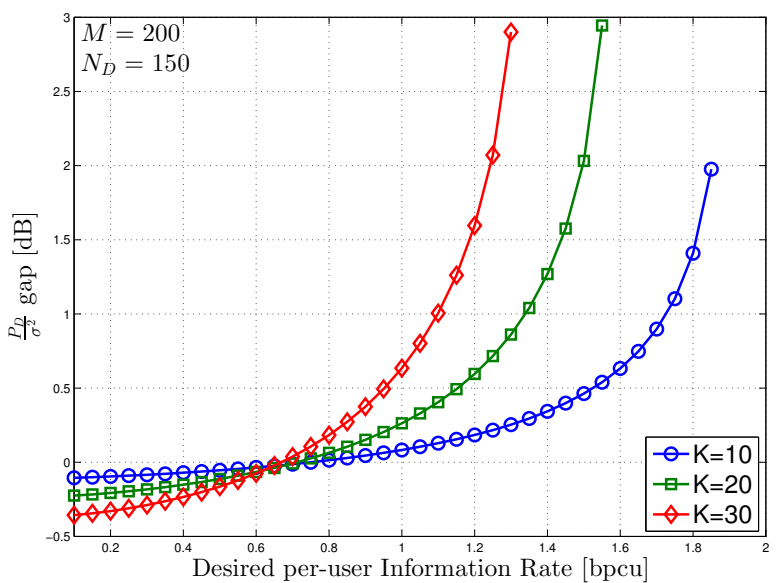

Fig. 2. $\frac{P_{D}}{\sigma^{2}}$ gap between $\mathrm{ZF}$ and $\mathrm{MRC}$ in $[\mathrm{dB}]$ to achieve a desired per-user information rate [bpcu], for fixed $M=200 \mathrm{BS}$ antennas, $N_{D}=150$ channel uses, $T_{s}=10^{-6} \mathrm{~s}$ and $c=4.7 \times 10^{-18}(\mathrm{rad} \mathrm{Hz})^{-1}$ for various values of the number of users, $K$.

achievable sum-rate expressions can provide additional insight on the effect of oscillator phase noise in Massive MIMO systems with ZF equalization.

\section{REFERENCES}

[1] G. Foschini and M. Gans, "On limits of wireless communications in a fading environment when using multiple antennas," Wireless Personal Communications, vol. 6, no. 3, pp. 311-335, Mar. 1998.

[2] D. Gesbert, et al., "Shifting the MIMO Paradigm," IEEE Sig. Proc. Mag., vol. 24, no. 5, pp. $36-46$, September 2007.

[3] T. L. Marzetta, "Noncooperative cellular wireless with unlimited numbers of base station antennas," IEEE Transactions on Wireless Communications, vol. 9, no. 11, pp. 3590-3600, Nov. 2010.

[4] F. Rusek, et al., "Scaling Up MIMO: Opportunities and Challenges with Very Large Arrays," IEEE Sig. Proc. Mag., vol. 30, no. 1, pp. 40-60, Jan. 2013.

[5] H. Q. Ngo, E. Larsson, and T. Marzetta, "Energy and spectral efficiency of very large multiuser MIMO systems," IEEE Transactions on Communications, vol. 61, no. 4, pp. 1436-1449, 2013.

[6] S. K. Mohammed and E. G. Larsson, "Single-User Beamforming in Large-Scale MISO Systems with Per-Antenna Constant-Envelope Constraints: The Doughnut Channel,' IEEE Trans. Wireless Comm., vol. 11, no. 11, pp. 3992-4005, Nov. 2012.

[7] J. Hoydis, S. ten Brink, and M. Debbah, "Massive MIMO in the UL/DL of Cellular Networks: How Many Antennas Do We Need?,' IEEE J. Sel. Areas Comm., vol. 31, no. 2, pp. 160-171, Feb. 2013.

[8] A. Pitarokoilis, S. K. Mohammed, and E. G. Larsson, "Effect of oscillator phase noise on the uplink performance of large MU-MIMO systems," in Proc. of the 50th Annual Allerton Conf. on Comm., Control and Computing, Monticello, IL, pp. 1190-1197, Oct. 2012.

[9] A. Demir, A. Mehrotra, and J. Roychowdhury, "Phase noise in oscillators: a unifying theory and numerical methods for characterization," IEEE Trans. on Circuits and Systems I: Fundamental Theory and Applications, vol. 47, no. 5, pp. 655-674, May 2000.

[10] D. Petrovic, W. Rave, and G. Fettweis, "Effects of phase noise on OFDM systems with and without PLL: Characterization and compensation," IEEE Trans. Comm., vol. 55, no. 8, pp. 1607 -1616, Aug. 2007.

[11] S. M. Kay, Fundamentals of statistical signal processing: estimation theory. Upper Saddle River, NJ, USA: Prentice-Hall, Inc., 1993.

[12] B. Hassibi and B. Hochwald, "How much training is needed in multipleantenna wireless links?," IEEE Trans. Inf. Theory, vol. 49, no. 4, pp. 951 - 963, Apr. 2003.

[13] T. L. Marzetta, "How much training is required for multiuser MIMO?," in Proc. 40th Asilomar Conf. on Sig., Sys. and Comp., pp. $359-363$, November 2006 